\title{
PRESSÃO ARTERIAL SISTÓLICA NOTURNA E DOENÇA CEREBROVASCULAR SUBCLÍNICA: ESTUDO SOBRE ANORMALIDADES CARDIOVASCULARES E LESÕES CEREBRAIS
}

\author{
NIGHT-TIME SYSTOLIC BLOOD PRESSURE AND SUBCLINICAL CEREBROVASCULAR \\ DISEASE: THE CARDIOVASCULAR ABNORMALITIES AND BRAIN \\ LESIONS (CABL) STUDY
}

Nakanishi K, Jin Z, Homma S, et al. Eur Heart J Cardiovasc Imaging. 2019 Jul 1;2O(7):765-771.

Comentador: Prof. Dr. José Fernando Vilela Martin

\section{RESUMO}

Introdução: Embora a pressão arterial (PA) ambulatorial seja um melhor preditor de desfechos cardiovasculares do que a pressão arterial de consultório, sua associação com a doença cerebrovascular subclínica não está esclarecida. Assim, esse estudo investigou a associação dos valores da PA de consultório e ambulatorial com a doença cerebrovascular subclínica em uma coorte populacional predominantemente idosa, sem histórico de acidente vascular cerebral prévio. Material e Métodos: 828 participantes foram submetidos à monitorização ambulatorial da PA por 24 horas (MAPA), ecocardiograma, ressonância magnética cerebral no estudo Cardiac Abnormalities and Brain Lesion (CABL). PA de vigília, PA durante o sono e de 24 horas, padrão de descenso noturno, elevação matutina (EM) e variabilidade da PA de 24 horas foram avaliados. A doença cerebrovascular subclínica foi definida como infarto cerebral silencioso (ICS) e volume de hiperintensidade da substância branca (VHSB). A associação das medidas da PA com a presença de ICS e o quartil superior do log-VHSB (log-VHSB 4) também foi analisada. Resultados: ICSs foram detectados em 111 pacientes (13,4\%). Na análise multivariável, apenas a PA sistólica (PAS) noturna esteve significantemente associada ao ICS [odds ratio (OR): 1,15 para cada $10 \mathrm{mmHg}, \mathrm{P}=0,042$ ], independente de fatores de risco cardiovascular e parâmetros ecocardiográficos avaliados. Embora PA na vigília, durante o sono, PA de 24 horas e o padrão não dipper tenham sido significantemente associados ao log-VHSB 4, a PAS noturna apresentou a associação mais forte (OR: 1,21 para cada $10 \mathrm{mmHg}, \mathrm{P}=0,003$ ) e foi o único preditor independente comparado aos outros parâmetros da PA. Medidas da PA de consultório, EM e variabilidade da PA não foram associadas à doença cerebrovascular subclínica nas análises ajustadas. Conclusões: A PAS elevada durante o sono apresenta forte associação com a doença cerebrovascular subclínica. O estudo conclui que a PAS noturna avaliada pela MAPA permite identificar indivíduos com maior risco de lesão cerebral hipertensiva.

\section{ANÁLISE CRÍTICA}

Esse estudo demonstrou que a PAS noturna foi um preditor mais forte da presença de doença cerebrovascular subclínica em uma amostra predominantemente idosa sem história de acidente vascular cerebral (AVC). A associação entre PAS durante o sono e doença cerebral subclínica ocorreu independente de outros fatores de risco cardiovascular tradicionais e de alterações ecocardiográficas relacionadas à hipertensão. ${ }^{1}$

Já se conhece que a PA obtida pela MAPA é melhor preditor de doenças cardiovasculares e de mortalidade do que a PA de consultório. ${ }^{2,3} \mathrm{O}$ primeiro relato desse achado data de 1988, quando O'Brien e colaboradores mostraram que pacientes hipertensos com menor queda noturna da PA cursavam com maior prevalência de AVC e os denominou de "não dippers". ${ }^{4}$ Desde então, vários estudos investigaram a associação entre a falta do descenso noturno (DN) e doenças cerebrovasculares. No entanto, os estudos sobre a presença de não dipper e risco de AVC tem apresentado resultados conflitantes. ${ }^{5-7}$

Em um estudo, a razão PAS sono/vigília não foi associada ao AVC em 7.458 indivíduos durante o acompanhamento médio de 9,6 anos. ${ }^{5}$ Outro estudo mostrou que a razão PAS sono/

1. Faculdade de Medicina de São José do Rio Preto (FAMERP), Livre-Docente em Cardiologia pela Universidade de São Paulo, São Paulo, Brasil 
vigília foi preditor da ocorrência de AVC em uma metanálise de 17.312 hipertensos. ${ }^{6}$ Nosso grupo avaliou a prevalência de DN em 163 indivíduos hipertensos e mostrou que a ausência de $\mathrm{DN}$ associou-se de maneira independente às lesões em órgãos-alvo analisadas - AVC e infarto do miocárdio. Embora a PAS no sono tenha sido maior no grupo não dipper, ela não se associou aos eventos avaliados. ${ }^{7}$

Entretanto, há evidências crescentes de que o nível médio da PA durante o sono, em vez do padrão noturno de não dipper, pode ser o maior preditor do risco de AVC em indivíduos com ou sem hipertensão. Fagard e colaboradores demonstraram que uma diferença de $17,6 \mathrm{mmHg}$ na PAS noturna foi associada a um aumento do risco de AVC em 3.468 hipertensos, enquanto a razão PAS sono/vigília não foi associada a AVC após o ajuste para PAS de 24 horas. $^{8}$

No presente estudo, os infartos cerebrais silenciosos (ICSs) e os volumes de hiperintensidade da substância branca (VHSBs), definidos por ressonância magnética cerebral, estão comumente presentes em adultos idosos e são importantes marcadores da doença cerebral de pequenos vasos. Embora os ICSs e VHSBs não estejam tipicamente ligados a sintomas evidentes de AVC clínico, eles não são totalmente silenciosos ou benignos, pois geralmente estão associados a sintomas neurológicos sutis e maior risco de AVC, comprometimento cognitivo, demência e morte. ${ }^{9-11}$

Vários mecanismos podem explicar a razão da PA noturna elevada se associar à doença cerebrovascular subclínica. Hiperatividade simpática persistente pode refletir melhor o estresse mecânico sobre a parede arterial do que a PA de vigília. ${ }^{12}$ Outros fatores podem estar presentes, entre eles, função endotelial prejudicada, ativação plaquetária e resposta inflamatória aumentada, e maior sensibilidade ao sal. ${ }^{13-15}$ Além disso, distúrbio do sono também pode estar envolvido na associação observada. ${ }^{16}$

Os autores também discutem sobre a elevação matutina (EM) da PA, mostrando que apesar de ser considerada um fenômeno fisiológico após o despertar, uma elevação acentuada e rápida pode se associar ao aumento do risco de doença cerebrovascular. Trabalhos têm demonstrado que EM elevada é preditor da incidência de AVC em pacientes hipertensos tratados $^{17}$ e nos indivíduos com EM $\geq 55$ mmHg comparados àqueles sem $\mathrm{EM}$, houve maior incidência de múltiplos ICSs. ${ }^{18}$ No presente estudo, a EM não foi significativamente associada à doença cerebral subclínica. Os autores justificam essa discrepância pelas diferentes populações e comorbidades avaliadas, uma vez que os asiáticos parecem ter maior PA matinal comparado com ocidentais.

A variabilidade ambulatorial da PA também foi avaliada no presente estudo e não demonstrou associação independente com doença cerebrovascular subclínica, sugerindo que valores médios elevados da PA, principalmente à noite, podem ser determinantes mais importantes da doença cerebral silenciosa do que flutuações da PA nas $24 \mathrm{~h}$.

Os autores também discutem a importância de acompanhamento mais próximo de indivíduos com PAS noturna elevada, uma vez que ICSs e VHSBs carregam um risco aumentado de AVC. Assim, terapia anti-hipertensiva com foco no período noturno para melhor controle da PA durante o sono, controle mais intensivo dos fatores de risco e tratamento para apneia do sono podem ser condutas úteis para reduzir a PAS noturna. A adoção dessas medidas pode exercer efeitos benéficos sobre a doença cerebrovascular subclínica ao longo do tempo.

\section{REFERÊNCIAS}

1. Nakanishi K, Jin Z, Homma S, et al. Night-time systolic blood pressure and subclinical cerebrovascular disease: the Cardiovascular Abnormalities and Brain Lesions (CABL) study. Eur Heart J Cardiovasc Imaging. 2019;20(7):765-71.

2. O'Brien E, Parati G, Stergiou G, et al. European Society of Hypertension position paper on ambulatory blood pressure monitoring. J Hypertens. 2013;31:1731-68.

3. Shimbo D, Abdalla M, Falzon L, Townsend RR, Muntner P. Role of ambulatory and home blood pressure monitoring in clinical practice: a narrative review. Ann Intern Med. 2015;163:691-700.

4. O'Brien E, Sheridan J, O'Malley K. Dippers and non-dippers. Lancet. 1988;2:397.

5. Boggia J, LiY, Thijs L, et al. Prognostic accuracy of day versus night ambulatory blood pressure: a cohort study. Lancet. 2007;370:1219-29.

6. Salles GF, Reboldi G, Fagard RH, et al. Prognostic effect of the nocturnal blood pressure fall in hypertensive patients: the ambulatory blood pressure collaboration in patients with hypertension $(\mathrm{ABC}-\mathrm{H})$ meta-analysis. Hypertension. 2016;67:693-700.

7. Vaz-de-Melo RO, Toledo JC, Loureiro AA, Cipullo JP, Moreno Júnior $\mathrm{H}$, Martin JF. Absence of Nocturnal Dipping is Associated with Stroke and Myocardium Infarction. Arq Bras Cardiol. 2010;94:79-85.

8. Fagard RH, Celis H, Thijs L, et al. Daytime and nighttime blood pressure as predictors of death and cause-specific cardiovascular events in hypertension. Hypertension. 2008;51:55-61.

9. Vermeer SE, Hollander M, van Dijk EJ, et al. Silent brain infarcts and white matter lesions increase stroke risk in the general population: the Rotterdam Scan Study. Stroke. 2003;34:1126-9.

10. Vermeer SE, Prins ND, den Heijer T, Hofman A, Koudstaal PJ, Breteler MM. Silent brain infarcts and the risk of dementia and cognitive decline. N Engl J Med. 2003;348:1215-22.

11. Wright $C B$, Dong $C$, Perez EJ, et al. Subclinical cerebrovascular disease increases the risk of incident stroke and mortality: the Northern Manhattan study. J Am Heart Assoc. 2017; 6:e004069.

12. Dodt C, Breckling U, Derad I, Fehm HL, Born J. Plasma epinephrine and norepinephrine concentrations of healthy humans associated with nighttime sleep and morning arousal. Hypertension. 1997;30:71-6.

13. Maio R, Perticone $M$, Sciacqua A, et al. Oxidative stress impairs endothelial function in nondipper hypertensive patients. Cardiovasc Ther. 2012;30:85-92.

14. Kaya MG, Yarlioglues M, Gunebakmaz O, et al. Platelet activation and inflammatory response in patients with non-dipper hyper-tension. Atherosclerosis. 2010;209:278-82.

15. Sachdeva A, Weder AB. Nocturnal sodium excretion, blood pressure dipping, and sodium sensitivity. Hypertension. 2006;48:527-33.

16. Portaluppi F, Provini F, Cortelli P, et al. Undiagnosed sleep-disordered breathing among male nondippers with essential hypertension. J Hypertens. 1997;15:1227-33.

17. Pierdomenico SD, Pierdomenico AM, Cuccurullo F. Morning blood pressure surge, dipping, and risk of ischemic stroke in elderly patients treated for hypertension. Am J Hypertens. 2014;27:564-70.

18. Kario K, Pickering TG, Umeda Y, et al. Morning surge in blood pressure as a predictor of silent and clinical cerebrovascular disease in elderly hypertensives: a prospective study. Circulation. 2003;107:1401-6. 